\title{
La relation de maître à disciple en question : transmission orale et écrite des savoirs divinatoires en Chine et à Taïwan
}

Challenging the Teacher-Student Relationship: Oral and Written Transmission of Divinatory Knowledge in China and Taiwan

師生傳承: 中國大陸及臺灣占卜知識的口授和文本式教學

\section{Stéphanie Homola}

\section{OpenEdition}

\section{Journals}

Édition électronique

URL : http://journals.openedition.org/extremeorient/276

DOI : 10.4000/extremeorient.276

ISSN : 2108-7105

Éditeur

Presses universitaires de Vincennes

Édition imprimée

Date de publication : 1 mai 2013

Pagination : 11-43

ISBN : 978-2-84292-367-9

ISSN : 0754-5010

Référence électronique

Stéphanie Homola, «La relation de maître à disciple en question : transmission orale et écrite des savoirs divinatoires en Chine et à Taïwan », Extrême-Orient Extrême-Occident [En ligne], 35 | 2013, mis en ligne le 01 mai 2016, consulté le 06 mai 2019. URL : http://journals.openedition.org/ extremeorient/276; DOI : 10.4000/extremeorient.276 


\title{
La relation de maître à disciple en question : transmission orale et écrite des savoirs divinatoires en Chine et à Taïwan
}

\author{
Stéphanie Homola
}

Longtemps condamnées comme «superstitions » par les régimes nationaliste et communiste, les pratiques de divination connaissent aujourd'hui une vague de popularité à Taïwan comme en Chine continentale. Elles ne bénéficient cependant d'aucune forme de reconnaissance institutionnelle. Pour lutter contre cette marginalisation, les spécialistes des arts divinatoires tentent d'adapter leurs savoirs à la société moderne. Comme dans tout processus de légitimation et de catégorisation des champs du savoir, la question de la transmission des connaissances et de l'enseignement est un enjeu central. Je propose ainsi de mettre en parallèle deux modes contrastés de transmission des savoirs mantiques à Taïwan et en Chine populaire, l'un privilégiant la transmission écrite, l'autre la transmission orale.

Cet article se concentre sur les pratiques divinatoires dites «déductives», regroupées sous l'appellation «arts divinatoires» (shushu). Cet ensemble comprend, parmi les techniques les plus répandues aujourd'hui, l'horoscopie calendaire (mingli), la physiognomonie, la chiromancie, l' analyse des caractères d'écriture (cezi), la géomancie (fengshui) ainsi que les méthodes divinatoires fondées sur le Livre des Mutations (zhanbu). Le processus de transmission de la Chine continentale à Taïwan que je décris par la suite concerne principalement les techniques sophistiquées de calcul du destin de l'horoscopie calendaire, telles que la méthode liuren, la méthode ziweidoushu et la méthode des «huit signes» (bazi). Avec les méthodes taiyi (du «grand Un») et qimen dunjia (du «cycle caché»), la méthode liuren (des «six troncs $r e n^{1} »$ ) forme les sanshi, trois procédés mantiques pratiqués par le Bureau astronomique pour le bénéfice de l'empereur et qui connaissent leur âge d'or sous la dynastie Song (9601279). À partir du VIII siècle, sous l'influence de l'astrologie grecque, hindoue et iranienne, la méthode taiyi se développe en une forme d'astrologie encore

1. Du nom de la neuvième composante du cycle des dix troncs célestes, voir p. 24. 
largement populaire aujourd'hui à Taïwan et connue sous le nom de ziweidoushu («Nombres du Boisseau (du Nord) dans (le palais de) la Sublimité pourpre»). La méthode des «huit signes », fondée sur l'analyse des composantes calendériques de la date de naissance d'un individu, prospère également sous les Song, à une époque où l'analyse du destin qui était jusqu'alors réservée à l'empereur se répand parmi le peuple ${ }^{2}$.

Les arts divinatoires se distinguent des pratiques de divination dites « intuitives » qui visent à obtenir des informations en communiquant avec les dieux ou les ancêtres au moyen de blocs de divination (jiao), de bâtons de divination (qian) ou par l'intermédiaire d'un médium. Ils se distinguent également du choix des jours fastes (zeri) qui consiste à consulter des almanachs pour déterminer les dates les plus propices à différentes entreprises (déménagement, voyage...).

À Taïwan, l'horoscopie calendaire s'est développée à partir du repli du régime nationaliste sur l'île en 1949 ainsi que sous l'influence du Japon qui a contribué en particulier à l'essor de la méthode d'horoscopie ziweidoushu. Dans ce contexte, il est intéressant de mettre en relation deux dimensions de la transmission des connaissances, comprise à la fois comme circulation des savoirs et comme méthode d'enseignement. Dans les trois premières parties de cet article, consacrées à la transmission et au développement de l'horoscopie à Taïwan, je propose ainsi d'étudier comment la circulation des hommes et des livres dans le temps et dans l'espace a affecté le mode de transfert des connaissances.

À partir des années 1980 , en réaction à la dégradation de la qualité de la transmission et à la multiplication des écoles de pensée, des spécialistes des arts divinatoires taïwanais entreprennent de reformuler et d'unifier les concepts et les techniques mantiques pour les adapter aux exigences rationnelles et scientifiques de la société contemporaine. Ils poursuivent en cela une entreprise de rationalisation qui a été initiée en Chine continentale à l'époque républicaine et qui se traduit, à Taïwan, par un essor des manuels de vulgarisation et une relative standardisation de la formation sous forme de cours collectifs. Les réformateurs tentent ainsi d'établir un nouveau paradigme de transmission des connaissances divinatoires en remplaçant le mode traditionnel d'enseignement fondé sur la relation de maître à disciple par un mode de transmission forgé sur le modèle universitaire.

En Chine continentale, depuis une dizaine d'années, la diffusion croissante de manuels de divination à destination du grand public semble également remettre en cause la relation de maître à disciple comme mode de transmission privilégié des arts divinatoires. Pourtant, en m'appuyant sur une enquête de terrain menée

2. Sur l'histoire de ces méthodes, voir Ho 2003. 
auprès de praticiens amateurs de Kaifeng, dans la province du Henan, entre 2009 et 2011, je propose de montrer, dans les deux dernières parties de cet article, qu'une véritable «économie» de la transmission orale reste vivante dans le cadre d'une relation de maître à disciple. L'enseignement des techniques s'accomplit ainsi sur le mode de l' «affinité prédestinée», du voyage initiatique et de la transmission orale des légendes et des savoir-faire.

L'étude de ces deux modes de transmission, l'un forgé sur le modèle universitaire, l'autre sur le modèle de la relation de maître à disciple, est menée à deux échelles différentes et s'appuie sur deux approches méthodologiques distinctes. D'un côté, je propose une analyse diachronique d'une tendance de fond d'adaptation du mode de transmission des savoirs divinatoires à la modernité, qui s'étend du début du $\mathrm{Xx}^{\mathrm{e}}$ siècle à aujourd'hui, depuis la Chine continentale jusqu'à Taïwan. Ainsi, pour comprendre les tentatives de formation d'un système d'enseignement de type universitaire à Taïwan, je m'intéresse aux spécialistes reconnus des arts mantiques et à leurs textes, à l'histoire de la transmission des corpus écrits et au développement des écoles de pensée.

D'un autre côté, je propose de nuancer ce mouvement général de rationalisation du transfert des connaissances divinatoires par le contre-exemple d'une étude de cas qui met en évidence l'importance de la relation personnelle et de l'oralité dans la transmission des savoirs. L'étude de la relation de maître à disciple à Kaifeng s'appuie ainsi principalement sur les récits oraux de praticiens amateurs et l'observation de leur pratique dans la vie quotidienne.

Il ne s'agit donc pas d'opposer de manière simplificatrice Taïwan à la Chine continentale mais d'étudier les caractéristiques de ces deux modes de transmission et les conditions particulières dans lesquelles ils s'inscrivent.

\section{Les vicissitudes de la transmission des savoirs divinatoires du continent à Taïwan}

Au début du $\mathrm{XX}^{\mathrm{e}}$ siècle, le mouvement révolutionnaire anti-traditionnaliste et anti-confucéen qui lance le processus de modernisation de la société chinoise se traduit par l'introduction dans le champ intellectuel puis politique des catégories occidentales modernes de science, religion et superstition. Cette catégorisation bouleverse le statut des personnes qui pratiquent la divination et les modes de transmission des savoirs mantiques.

Dans la société impériale traditionnelle, la majorité des rites de ce que l'on peut appeler la «religion chinoise» (tels que le culte aux ancêtres, aux dieux locaux...) est effectuée au sein des communautés par ses membres eux-mêmes. Pour certains rituels (enterrement, inauguration d'un temple...), les communautés font appel à l'un ou l'autre des spécialistes des trois formes institutionnalisées de 
la religion chinoise que sont le bouddhisme, le taoïsme et le confucianisme. Des membres de la communauté peuvent aussi s'initier à certaines pratiques inspirées des trois enseignements afin de rendre directement service à la communauté. C'est le cas des devins qui exercent de manière professionnelle ou occasionnelle. Ils sont issus de lignées héréditaires de praticiens ou se forment auprès de maîtres locaux ou itinérants. Par ailleurs, le Livre des Mutations faisant partie des Classiques confucéens au programme des concours de recrutement des fonctionnaires impériaux, les lettrés confucéens se passionnent pour les «études du Livre des Mutations » (yixue) et pratiquent une forme élitiste de divination fondée sur la maîtrise de ce classique.

À la disparition de l'Empire, sont reconnues comme religions officielles, le bouddhisme, le taoïsme, le christianisme, le protestantisme et l'islam. En cohérence avec le concept occidental de religion, ces religions institutionnelles sont comprises comme des communautés exclusives de fidèles regroupés autour d'un clergé. Dès lors, les cultes et rituels religieux qui étaient assurés par les communautés, dénués de tout statut, sont qualifiés de «superstitions » et combattus comme tels. Le confucianisme, lui, indissociable du système impérial, perd toute structure institutionnelle: les anciens lettrés confucéens, représentants de l'élite intellectuelle, deviennent hommes politiques, professeurs d'université ou érudits locaux.

Le mouvement anti-impérialiste et anti-religieux du 4 mai 1919 marque une radicalisation des politiques de modernisation. Entre 1928 et 1930, diverses campagnes d'éradication de la superstition (pochu mixin yundong) ${ }^{3} \mathrm{~s}$ 'attaquent directement aux devins.

Ces mesures se soldent pourtant par un échec. Les pratiques de divination restent très populaires sous la République et donnent lieu à de nombreuses publications sur la géomancie, la méthode des «huit signes » ou le choix des jours. Certains spécialistes jouissent d'une grande renommée, à l'instar des «Trois grands maîtres de Shanghai»: Yuan Shushan, Xu Lewu et Wei Qianli. Les autorités découvrent en effet que les devins savent s'organiser pour protester et revendiquer leur place dans la nouvelle société créée par le Kuomintang. Tout d'abord, ils s'approprient la rhétorique nationaliste et le «principe de bien-être du peuple» (minshengzhuyi) de Sun Yat-sen pour revendiquer le droit à gagner leur vie selon leurs moyens. Ils bénéficient également du soutien des élites politiques et économiques, notamment shanghaiennes, qui ont fréquemment recours à leurs

3. «Procédures pour l'abolition des métiers de la divination, de l'astrologie, de la physiognomonie, de la chiromancie, de la sorcellerie et de la géomancie» (1928), «Interdiction des médecines divinatoires» (1929), «Procédures pour l'interdiction et la gestion des objets et des métiers de la superstition» (1930). Voir Duara 1991 : 79-80. 
services. Enfin, pour défendre les arts mantiques face aux campagnes contre les superstitions, des spécialistes tels que Yuan Shushan, entreprennent de les adapter à la nouvelle idéologie modernisatrice ${ }^{4}$.

De même que les institutions bouddhistes et taoïstes s'efforcent de conformer leur dogme et leur organisation au nouveau concept de religion, Yuan réalise l'urgence d'établir une synthèse des connaissances divinatoires pour répondre au défi posé par la science occidentale, perçue comme systématique et impersonnelle ${ }^{5}$. Tout d'abord, il prône une analyse critique des sources anciennes et ouvrages de référence afin de remédier à leurs travers (exemples sans commentaires ou commentaires sans exemples, foisonnement et incohérence des théories, passages verbeux ou trop généraux...) et d'en réaliser une synthèse. Il développe ainsi dans ses écrits une approche innovante combinant les explications des techniques, de larges références aux textes classiques et de nombreux exemples historiques à l'appui des théories. En établissant des références croisées systématiques entre différents arts divinatoires, il contribue également à l'unification des «études sur le destin» (lumingxue).

Une autre voie de réforme proposée par Yuan nous intéresse particulièrement ici. Poursuivant une réflexion entamée dès la fin du XIX ${ }^{\mathrm{e}}$ siècle par les réformateurs chinois, il prône le rejet de la transmission de maître à disciple comme méthode d'enseignement des arts divinatoires: «The appeal to abandon the traditional ways of transmission from "teacher to student" (shisheng guanxi) was one of the predominant definitions of "science" in Late Imperial and Republican China.${ }^{6} \gg$ Ce modèle traditionnel de transfert des connaissances est fondé sur la relation personnelle entre le maître et son disciple. L'enseignement individuel et la constitution d'écoles ou de lignées permettent de contrôler le processus de transmission et de protéger les intérêts de la profession. La formation n'est pas uniquement technique: elle exige du disciple qu'il habite chez son maître, partage son mode de vie et le serve au quotidien. La relation s'inscrit aussi dans la durée: le maître ne révèle que progressivement son savoir pour éprouver la motivation et les capacités du disciple. Enfin, l'accent est mis sur la transmission orale et l'apprentissage par cœur des formules et techniques.

Au contraire, les réformateurs des arts mantiques soutiennent que les théories divinatoires, une fois uniformisées, synthétisées et systématisées, peuvent être appliquées de manière fixe, telles des lois scientifiques, et donc être transmises

4. Voir Lackner 2012 qui constitue une étude préliminaire à une biographie de Yuan Shushan.

5. Comme le note également M. Lackner, dans ce contexte, c'est par rapport à la science et non à la religion que les arts mantiques se positionnent.

6. Lackner 2012: 13. 
dans des livres et non plus par des maîtres. Dans cette perspective, l'enseignement peut devenir collectif. Ainsi, en 1930, Yuan Shushan est le premier praticien à faire de la publicité dans les journaux de Shanghai pour proposer des cours sur les techniques de divination, d'abord par correspondance puis à des classes d'étudiants ${ }^{7}$. Il est suivi par de nombreux maîtres dans les années 1930 et c'est ce système d'enseignement de la divination qui perdure encore aujourd'hui à Taïwan.

En effet, alors que sur le continent le régime communiste tente d'éradiquer les pratiques superstitieuses, l'entreprise de rationalisation de l'horoscopie initiée par Yuan se poursuit à Taïwan à partir des années 1980 .

Les méthodes de divination fondées sur le Livre des Mutations et les techniques sophistiquées d'horoscopie calendaire comme la méthode des « huit signes », sont très peu pratiquées à Taïwan avant 1949 et sont introduites sur l'île par les Chinois réfugiés du continent après la guerre civile. De manière ironique, c'est le repli du régime nationaliste, pourtant farouchement opposé aux pratiques «superstitieuses », qui a favorisé le développement de l'horoscopie à Taïwan.

Jusqu' au XIX ${ }^{\mathrm{e}}$ siècle, les populations d'origine chinoise à Taïwan, issues des vagues migratoires en provenance des provinces du Fujian et du Guangdong, pratiquent principalement des rituels de divination dans les temples au moyen de blocs et de bâtons de divination et le choix de jours auspicieux à l'aide d'almanachs ${ }^{8}$.

Le modèle japonais ayant fortement influencé la réflexion des intellectuels chinois sur la modernisation de la société ainsi que les politiques républicaines, il n'est guère surprenant que le régime colonial japonais (1895-1945) ait mené à Taïwan des politiques de lutte contre les superstitions similaires à celles conduites sur le continent. Une étude publiée en 1939 sur «La religion et les mauvaises habitudes superstitieuses à Taïwan ${ }^{9}$ » décrit ainsi les «coutumes anormales » (biantaifengsu) des géomanciens. Un praticien taïwanais contemporain explique ainsi pourquoi l'horoscopie était si peu répandue à cette époque : «À l'époque où Taïwan était sous contrôle japonais, le Japon interdisait l'importation de livres depuis le continent tels que les almanachs, les calendriers et les livres d'horoscopie, si bien que peu de personnes étudiaient l'horoscopie et qu'il y avait peu de spécialistes à Taïwan. ${ }^{10}{ }^{»}$

7. Foo 2009.

8. Feuchtwang 1974 : 277. L'auteur mentionne ainsi un rituel de divination accompli dans un temple à la suite d'un incendie pour connaître la raison de la colère du dieu.

9. Sô Keirai 1939.

10. Zhong Yiming 1995. L'auteur signale l'ouvrage de Bai Huiwen comme le seul livre d'horoscopie disponible à Taïwan à cette époque. Cet ouvrage est répertorié dans le 
Pendant et après la guerre civile, les spécialistes des arts divinatoires, souvent proches des milieux nationalistes, fuient la Chine populaire, certains vers HongKong, d'autres vers Taïwan, comme Yuan. Parmi les troupes nationalistes et les civils qui se réfugient à Taïwan se trouvent également des praticiens professionnels et amateurs qui emmènent avec eux des éditions hongkongaises des livres de Yuan, Xu et Wei. La première édition taïwanaise d'un ouvrage d'horoscopie semble être une réédition d'un manuel de Xu à Taipei en $1959^{11}$.

C'est avec ces ouvrages et auprès des Chinois réfugiés du continent (waishengren) que se forme une première génération d'horoscopistes taïwanais (bendiren).

\section{La formation d'une première génération d'horoscopistes à Taïwan et l'essor de la méthode ziweidoushu}

Trois phénomènes caractérisent le développement des arts divinatoires à Taïwan : l'introduction de la méthode d'astrologie calendaire ziweidoushu; un engouement populaire qui se traduit par un essor des publications ; la prolifération d'écoles de pensée autour de la méthode ziweidoushu.

Avant 1949, la méthode ziweidoushu était pratiquée à Shanghai et à HongKong mais était beaucoup moins répandue que les autres formes d'horoscopie. À Taïwan, un Chinois du continent, Chen Yueqi, est connu pour avoir ramené avec lui un exemplaire de l'édition xylographique du Doushu quanji (Euvres complètes de la méthode des Nombres du Boisseau du Nord), un ouvrage classique de référence de la méthode ziweidoushu, datant de la $9^{\mathrm{e}}$ année de l'empereur Tongzhi des Qing (1870) qu'il fait republier à Taipei en 1966. Mais ce sont surtout des ouvrages en provenance du Japon qui vont jouer un rôle déterminant dans l'essor de cette technique à Taïwan ${ }^{12}$.

En 1966, un vétéran des troupes nationalistes, Zhang Yaowen, effectue un voyage d'étude au Japon. Il se lie d'amitié avec des spécialistes japonais des arts divinatoires, Abe Taizan et surtout Satô Rikuryû qui lui enseigne diverses techniques dont la méthode ziweidoushu. Il rentre à Taïwan en 1967 où il fonde une école de pensée appelée «école de la transparence» (toupai). Ainsi, outre l'édition du Doushu quanji évoquée plus haut, les livres de ziweidoushu les plus anciens que j'ai retrouvés à Taïwan sont deux ouvrages de 1968, l'un

catalogue du National Digital Archives Program où il est classé dans la catégorie «Bibliothèque de la résidence du gouverneur de Taïwan». Voir Bai Huiwen 1936.

11. Xu Lewu 1959.

12. Des ouvrages d'astrologie à l'origine de la forme moderne de la méthode ziweidoushu ont été introduits au Japon dès le IX ${ }^{\mathrm{e}}$ siècle. Voir Ho 2003: 69-73. 
compilé et publié au Japon par Satô et l'autre, publié en chinois à Taïwan par Abe $^{13}$. En 1970, Zhang publie le premier livre de ziweidoushu d'un auteur chinois à Taïwan ${ }^{14}$. Par la suite, il compile des livres de Satô notamment dans une collection en cinq tomes qui reprend une grande diversité de techniques: horoscopie, divination par le Livre des Mutations, physiognomonie, médecine traditionnelle et fengshui ${ }^{15}$. Parallèlement et surtout à partir du début des années 1980, Zhang publie aussi ses propres ouvrages sur les arts divinatoires sans référence à Satô.

Le deuxième phénomène marquant est l'engouement que suscitent les arts divinatoires, et en particulier la méthode ziweidoushu, à Taïwan à partir des années 1980. Cela s'explique tout d'abord par un contexte politique favorable d'affaiblissement du discours officiel de dénigrement des pratiques populaires. En effet, l'opposition politique entre les régimes communiste et nationaliste chinois pousse ce dernier à réévaluer son héritage historique et, face aux destructions de la Révolution Culturelle sur le continent (1966-1976), à se poser en garant de la culture traditionnelle chinoise ${ }^{16}$. Dès lors, le régime amorce un tournant dans sa politique religieuse: les superstitions sont peu à peu réévaluées puis deviennent objet d'étude sous le nom de «religion populaire». De plus, à partir de la fin des années 1980 , le processus de démocratisation se traduit par un essor de l'industrie culturelle et du secteur de l'édition qui profite notamment aux publications sur les arts divinatoires.

Pour essayer d'évaluer l'ampleur des publications, j' ai mené une recherche systématique par mots-clés dans le catalogue de la Bibliothèque Nationale de Taïwan. Le graphique ci-dessous représente la répartition des 1395 publications classées dans la catégorie «livres de divination» (mingshu) entre 1980 et 2011. Le graphique fait apparaître deux hausses des publications, une première au milieu des années 1980 et une deuxième, plus importante en valeur absolue, au milieu des années $1990{ }^{17}$.

13. Satô Fumio et Satô Rikuryû 1968; Abe Taizan 1968.

14. Zhang Yaowen 1970.

15. Satô Rikuryû et Zhang Yaowen 1975.

16. Il est ainsi remarquable que, malgré le rôle du Japon dans la transmission et le développement des arts divinatoires à Taïwan, les touristes japonais se rendent aujourd'hui dans les «rues de la divination» à Taipei pour retrouver des pratiques considérées comme plus «authentiques», du fait de l'origine chinoise, certes lointaine, de la divination japonaise mais aussi de l'image de conservatoire du patrimoine chinois dont bénéficie Taïwan par contraste avec la Chine continentale.

17. Cette recherche a été réalisée en décembre 2011. La recension de 1395 ouvrages est loin d'être exhaustive et dépend notamment du classement des bibliothécaires. Une 
Tableau 1. Nombre de publications sur la divination à Taïwan entre 1980 et 2011

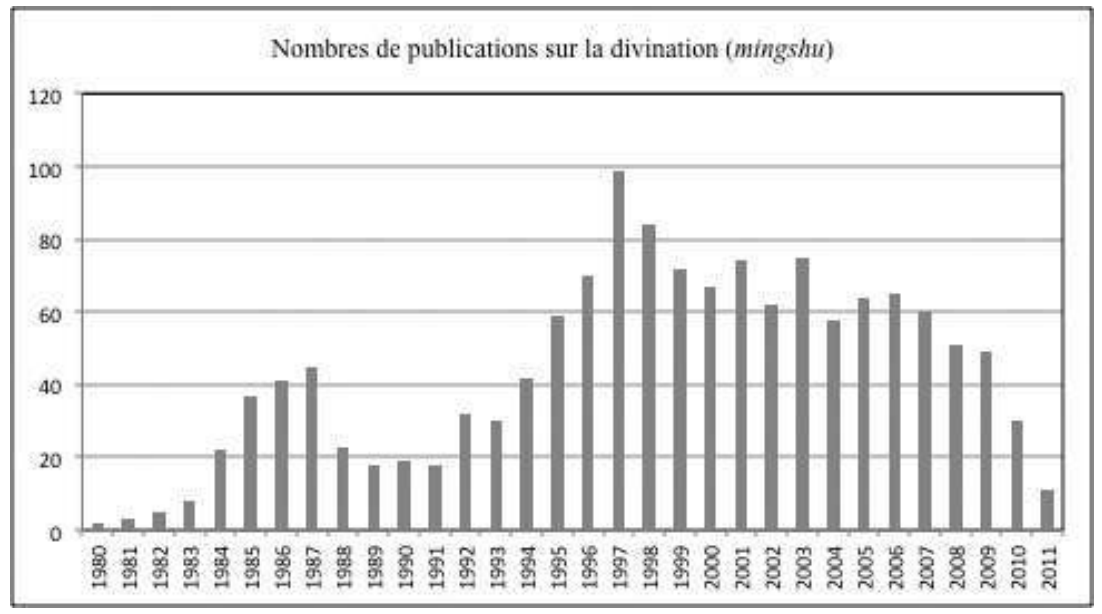

Comment expliquer ces deux vagues de publications? D'après mes recherches, la vague du milieu des années 1980 correspond aux publications de la première génération de praticiens taïwanais qui s'est formée dans les années 1960 et 1970 auprès de Chinois du continent (spécialistes réfugiés à Taïwan comme Yuan, amateurs éclairés, moines) et de maîtres japonais, soit directement à leur contact soit par l'intermédiaire de leurs ouvrages. Comme le montre le parcours de Zhang Yaowen, étant donné la complexité des techniques, une vingtaine d'années a été nécessaire pour que des praticiens nés à Taïwan puissent les assimiler et les transmettre à leur tour à travers leurs écrits. Le nombre de publications de cette première vague, relativement faible en valeur absolue, correspond à une diffusion des ouvrages parmi les praticiens spécialistes des arts divinatoires.

L'ampleur de la deuxième vague du milieu des années 1990 (presque cent ouvrages publiés en 1997) suggère cette fois une diffusion auprès d'un public plus large, non seulement de praticiens professionnels et amateurs, mais également de clients. À cette époque, le développement médiatique et marchand de la divination autour des publications, des consultations et des cours, a été qualifié de «fièvre de la divination» (suanming re).

Quant au troisième phénomène, la prolifération d'écoles de pensée autour de la méthode ziweidoushu, il s'agit d'un développement récent apparu dans les

recherche portant sur les 404 publications contenant «doushu» dans le titre entre 1980 et 2011 fait apparaître une répartition similaire. 
années 1980, bien que les maîtres de ces écoles revendiquent leur appartenance à des lignées anciennes s'étendant sur plusieurs générations. Ainsi, après avoir étudié auprès d'Abe Taizan et Satô Rikuryû et diffusé leurs livres à Taïwan, Zhang Yaowen s'est présenté comme le grand maître de la $13^{\text {e }}$ génération de l'école toupai, qui aurait été fondée à l'époque Ming. Chen Yueqi, à l'origine de la première publication à Taïwan du Doushu quanji en 1966, s'est présenté dans les années 1980, en s'appuyant notamment sur son nom, comme le grand maître de l' «école de la confirmation de l'oracle» (zhanyan pai) et unique descendant de la $41^{\mathrm{e}}$ génération de Chen Xiyi, inventeur légendaire de la méthode ziweidoushu.

Des particularités revendiquées par chaque doctrine se dégagent, paradoxalement, des mécanismes communs de formation d'une école (pai). Tout d'abord, le prestige d'une école se compte en générations de disciples. Le disciple-fondateur moderne s'applique ainsi à reconstruire l'arbre généalogique en remontant le plus loin possible dans le temps. Pour asseoir la singularité de la lignée, il est courant de choisir un ancêtre différent de la tradition dominante (en l'occurrence Chen Xiyi), parmi les nombreux personnages taoïstes et érudits de l'histoire chinoise de l'époque Tang et Song, grandes périodes de développement de l'horoscopie calendaire. Autre élément essentiel dans la construction de l'identité d'une école, la possession d'un manuel ancien et rare prouve l'appartenance à la lignée du disciple-fondateur moderne. Le secret de la transmission, quant à lui, permet de justifier pourquoi le livre ne refait surface qu'au milieu des années 1980. Les disciples-fondateurs s'appliquent alors à collecter, éditer et commenter les ouvrages secrets de l'école. L'incohérence voire la trahison que représente la révélation de techniques secrètes conservées si longtemps est rarement évoquée. Enfin, chaque école se distingue par l'adoption d'une variante technique ou d'une interprétation singulière de la méthode ziweidoushu. Les ouvrages de ces différentes écoles, volontiers ésotériques et consacrés aux conceptions personnelles du maître, constituent une bonne part des livres publiés dans les années 1990.

Trois raisons peuvent être avancées pour expliquer que le monde du ziweidoushu ait cristallisé ce phénomène de foisonnement d'écoles. Tout d'abord, la complexité de la méthode et les multiples influences dont elle témoigne (chinoises, grecques, babyloniennes, hindoues, iraniennes, japonaises) offrent un grand choix de références et de particularités techniques aux praticiens qui souhaitent se singulariser. Ensuite, le caractère marginal de cette technique jusqu'à la moitié du $\mathrm{xx}^{\mathrm{e}}$ siècle n'a pas favorisé la formation d'un consensus autour de procédés et de textes de référence parmi les praticiens, contrairement à d'autres méthodes d'horoscopie telles que la méthode des « huit signes ». Enfin, comme nous le verrons par la suite, bien que des recherches académiques aient 
été menées sur l'histoire de la méthode ziweidoush ${ }^{18}$, le cloisonnement entre le monde des praticiens et celui de la recherche ne favorise pas le partage des savoirs.

À partir des années 1980, c'est en réaction à cette multiplication des écoles de pensée et des publications qu'ils considèrent de médiocre qualité, que des praticiens préconisent une modernisation des arts divinatoires et de leur mode de transmission.

\section{Du maître au professeur : vers un nouveau mode d'enseignement des arts divinatoires}

\section{Critique des écoles de pensée et du mode de transmission de maître à disciple}

Contrairement à Yuan Shushan qui, à son époque, craignait pour la survie même des arts divinatoires, ces praticiens réformateurs font face à un foisonnement anarchique et incohérent de livres, de savoirs et de pratiques. Bien qu'ils soient souvent regroupés sous l'appellation d' "école moderne » (xiandai pai), ces praticiens refusent de s'inscrire dans une lignée. Ils critiquent ainsi la multiplication absurde des écoles, le culte de la personnalité et l'atmosphère magique qui entourent les maîtres et le soi-disant secret de la transmission des techniques. Ils déplorent que les maîtres de ces écoles, qui ont souvent reçu une formation superficielle du fait de la transmission chaotique des savoirs divinatoires du continent à Taïwan, véhiculent à travers leurs publications des conceptions irrationnelles des arts mantiques et en compromettent ainsi l'institutionnalisation.

Le rejet des écoles par ces praticiens s'accompagne également d'une critique de la relation maître-disciple, amorcée au début de l'ère républicaine où ce mode de transmission était considéré comme un obstacle à l'adaptation des savoirs traditionnels à la modernité. Ainsi, si les praticiens de l' «école moderne», comme la plupart des spécialistes contemporains, ont été initiés aux arts divinatoires par des maîtres originaires du continent, ils remettent largement en cause ce type de formation. C'est le cas de Ruli Jushi, un praticien de la région de Taipei dont je suis les activités d'enseignement et de publication depuis 2007 et qui a été le disciple d'un moine bouddhiste. Ce dernier, un ancien commandant des troupes nationalistes réfugiées à Taïwan, a quitté la carrière militaire pour vivre dans un monastère de Hong-Kong où, devenu moine, il s'est instruit dans les arts mantiques. Lorsque Ruli Jushi l'a rencontré en 1991 à Taïwan, il était âgé

18. Ho 2003. 
de 90 ans. Ruli Jushi est devenu officiellement son disciple (baishi) et le maître l'a initié au Livre des mutations jusqu'à sa mort trois ans plus tard. Pour Ruli Jushi, cette initiation a davantage été d'ordre spirituel que technique. En effet, même s'il reconnaît les talents de son maître, Ruli Jushi avoue n'avoir pas appris grand-chose pendant ces trois années qu'il a surtout passées à s'occuper du vieillard. Il insiste sur la nécessité d'apprendre par soi-même pour comprendre le véritable sens de la divination. C'est en lisant une grande quantité de livres de référence qu'il s'est formé lui-même à différentes techniques divinatoires ${ }^{19}$.

\section{Rationalisation et vulgarisation des arts divinatoires}

Les praticiens de l'«école moderne» reformulent les savoirs divinatoires pour les adapter aux avancées de la science et de la société contemporaine. Cette reformulation est nécessaire pour combattre les pratiques actuelles des charlatans (incarnés par les écoles), mais aussi pour réformer les techniques traditionnelles qui, par leur manque de rigueur, ont permis les mésinterprétations actuelles. Sans entrer dans les détails, il est possible de dégager les principes de cette entreprise de rationalisation qui vise à démarquer l'horoscopie de conceptions déterministes et magiques et de la croyance dans les esprits.

Tout d'abord, à l'instar de Yuan Shushan dans l'entre-deux-guerres, les réformateurs prônent un retour aux sources. Ainsi, pour être capable de juger par lui-même et ne plus être tributaire des interprétations embrouillées et irrationnelles de ses contemporains, Ruli Jushi a entrepris une relecture critique de tous les textes fondamentaux des arts divinatoires depuis le Lunheng (Balance des discours) de Wang Chong. En sélectionnant les ouvrages de référence, en retraçant et en évaluant les apports de chacun sur différents concepts fondamentaux, la démarche de Ruli Jushi contribue à une standardisation de la discipline.

Par ailleurs, le concept de destin est reformulé selon une conception non déterministe qui limite le rôle de l'horoscope dans la destinée d'une personne au profit des facteurs de l'environnement extérieur. Toujours pour se démarquer d'une conception déterministe, les réformateurs prônent une interprétation relativiste de l'horoscope : une personne ne peut être évaluée en elle-même mais en fonction des situations dans lesquelles elle se trouve. Elle est caractérisée

19. David Kim note un phénomène semblable dans son étude sur les chamans-devins dans la société coréenne contemporaine : contrairement à ce que l'on peut lire dans la littérature folkloriste, les chamans sont loin d'entretenir une relation à vie avec le chaman qui les a initiés. Une fois seuls, ils doivent s'appuyer sur d'autres sources de connaissance comme la littérature académique ou des récits de fiction pour parfaire leur pratique. Voir Kim 2009: 76. 
non par une identité unique mais par de multiples identités qui s'actualisent selon les circonstances.

Enfin, les réformateurs insistent sur le caractère non magique de la divination. L'horoscopie est un produit de la statistique qui permet d'évaluer la personnalité d'un individu et de le conseiller dans son adaptation à son environnement. Ainsi, les différents éléments constitutifs de l'horoscope ne doivent pas être interprétés littéralement. L'horoscope est une représentation virtuelle du temps et de l'espace qui n'a qu'un sens symbolique. Toute référence aux esprits est éliminée et le nombre d'étoiles est réduit et ordonné. Par exemple, les étoiles dites «auspicieuses» (ji) et «néfastes » $(s h a)$ ne sont en elles-mêmes ni positives ni négatives et doivent être interprétées dans leurs relations les unes avec les autres. Les spécialistes contemporains considèrent même que les étoiles néfastes indiquent des prédispositions dans le domaine de la haute technologie.

Cette entreprise de rationalisation combinée à une diffusion à grande échelle a entraîné un phénomène de vulgarisation. Cet élargissement de l'accès aux savoirs divinatoires est symbolisé par l'essor de manuels didactiques qui a contribué à la vague de publications du milieu des années 1990. En effet, les ouvrages anciens de divination ne visaient pas à expliquer les méthodes de manière exhaustive et servaient principalement aux initiés comme mémentos techniques. Souvent incomplets, rédigés en langue classique et dans un langage volontairement ésotérique sous forme de poèmes rimés, ils n'avaient pas vocation à se substituer à l'enseignement oral du maître. Quant aux livres contemporains des écoles de pensée évoqués plus haut, ils visent moins à faire comprendre les techniques qu'à renforcer le prestige du maître et à accroître le nombre de ses adeptes. À l'inverse, rédigés en langue vernaculaire (baihua) et dans un langage simple, les manuels de l' «école moderne» ont l'objectif affiché de se substituer aux maîtres. Ils rendent les techniques accessibles à un public de non-spécialistes, soit aux apprentis praticiens qui peuvent s'initier et se perfectionner grâce à des manuels clairs et didactiques, soit aux clients même des devins qui font un usage domestique des techniques divinatoires et qui ne sont pas prêts à s'investir dans des années de formation. Les clients peuvent ainsi mener leur propre consultation grâce aux manuels de la veine «how-to» (buqiuren) sur des thématiques aussi diverses que le mariage, l'argent ou la maladie. Certains, développant un intérêt particulier, peuvent être amenés à conseiller leur entourage et à devenir euxmêmes praticiens. Cette vulgarisation des savoirs mantiques tend ainsi à brouiller les catégories entre spécialistes professionnels, amateurs et usagers des arts divinatoires.

Dans ces manuels, l'usage de tableaux à la place des poèmes ou formules rimées ( gejue ou koujue) est un exemple frappant de la manière dont le discours de modernisation de l'horoscopie se traduit très concrètement dans le mode 
d'enseignement des techniques. Dans les méthodes des «huit signes» et ziweidoushu, la première étape du calcul de l'horoscope consiste à traduire la date de naissance de l'individu du calendrier républicain (ou grégorien) dans le calendrier traditionnel luni-solaire, selon le cycle sexagésimal formé de la combinaison en binômes des dix troncs célestes (tiangan) et des douze branches terrestres (dizhi). Chaque paramètre de la date de naissance (année, mois, jour, heure) est ainsi représenté par un binôme tronc-branche, appelé également «pilier» $(z h u)$. Ce sont les «huit signes» (bazi) ou «quatre piliers» (sizhu) qui ont donné son nom à cette méthode d'horoscopie calendaire. Ainsi, un poème rimé intitulé « déterminer l'heure à partir du jour ${ }^{20}$ » aide le praticien à calculer le binôme correspondant à l'heure de naissance à partir du tronc du jour de naissance:

Jia ji qi jia zi

Yi geng qi bing $z i$

Bing xin qi wu zi

Ding ren qi geng zi

Wu gui qi ren zi.

[Les jours de tronc] jia ou ji commencent par [le binôme de l'heure] jia zi

[Les jours de tronc] yi ou geng commencent par [le binôme de l'heure] bing zi

[Les jours de tronc] bing ou xin commencent par [le binôme de l'heure] wu zi

[Les jours de tronc] ding ou ren commencent par [le binôme de l'heure] geng zi

[Les jours de tronc] wu ou gui commencent par [le binôme de l'heure] ren zi.

La première ligne du poème s'interprète ainsi: si le tronc du jour est jia ou $j i$, commencer à compter la première heure du jour (de $23 \mathrm{~h}$ à $1 \mathrm{~h}$ ) à partir du binôme jia zi, si bien que l'on compte ensuite, selon l'ordre fixe des binômes dans le cycle sexagésimal, que la deuxième heure est yi chou, la troisième bing yin, etc., jusqu'à atteindre l'heure de naissance considérée. L'ensemble des combinaisons comprises (mais non entièrement énoncées) dans ce poème peut être traduit sous forme de tableau. Seules les deux premières lignes du tableau (grisées) sont énoncées dans le poème.

20. Ruli Jushi 2010: 106. 
Tableau 2. «Déterminer l'heure du partir du jour ${ }^{21}$

\begin{tabular}{|c|c|c|c|c|c|}
\hline $\begin{array}{l}\text { Tronc du jour } \\
\text { Heure de } \\
\text { naissance }\end{array}$ & jia ji 甲己 & yi geng 乙庚 & bing xin 丙辛 & ding ren 丁壬 & wu gui 戊癸 \\
\hline 23h-1h zi 子 & jia zi 甲子 & bing zi 丙子 & wu zi 戊子 & geng zi 庚子 & ren zi 壬子 \\
\hline 1h-3h chou \# & yi chou 乙丑 & ding chou 丁丑 & ji chou 己丑 & xin chou 辛丑 & gui chou 癸丑 \\
\hline 3h-5h yin 寅 & bing yin 丙寅 & wu yin 戊寅 & geng yin 庚寅 & ren yin 壬寅 & jia yin 甲寅 \\
\hline 5h-7h mao 卯 & ding mao 丁卯 & ji mao 己卯 & xin mao 辛卯 & gui mao 癸卯 & yi mao 乙卯 \\
\hline 7h-9h chen 辰 & wu chen 戊辰 & geng chen 庚辰 & ren chen 壬辰 & jia chen 甲辰 & bing chen 丙辰 \\
\hline 9h-11h si 巳 & ji si 己巳 & xin si 辛巳 & gui si 癸巳 & yi si 乙巳 & ding si 丁巳 \\
\hline $11 \mathrm{~h}-13 \mathrm{~h} \mathrm{wu}$ 午 & geng wu 庚午 & ren wu 壬午 & jia wu 甲午 & bing wu 丙午 & wu wu 戊午 \\
\hline 13h-15h wei 未 & xin wei 辛未 & gui wei 癸未 & yi wei 乙未 & ding wei 丁未 & ji wei 己未 \\
\hline $15 \mathrm{~h}-17 \mathrm{~h}$ shen 申 & ren shen 壬申 & jia shen 甲申 & bing shen 丙申 & wu shen 戊申 & geng shen 庚申 \\
\hline 17h-19h you 西 & gui you 癸西 & yi you 乙酉 & ding you 丁酉 & ji you 己酉 & xin you 辛西 \\
\hline $19 \mathrm{~h}-21 \mathrm{~h} x$ 戊 & jia xu 甲戌 & bing $x u$ 丙戌 & wu xu 戊戌 & geng xu 庚戌 & ren xu 壬戌 \\
\hline $21 \mathrm{~h}-23 \mathrm{~h}$ hai 亥 & yi hai 乙亥 & ding hai 丁亥 & ji hai 己亥 & xin hai 辛亥 & gui hai 癸亥 \\
\hline
\end{tabular}

Les formules rimées correspondent au modèle de la transmission orale. Leur style condensé et volontairement ésotérique nécessite les explications d'un maître et elles ne sont donc compréhensibles que des initiés. De plus, elles permettent de retrouver rapidement l'ensemble des cas par le calcul mental, ce qui, d'un côté, réduit l'effort de mémorisation mais, d'un autre côté, nécessite une grande pratique pour assimiler et manipuler les différents paramètres. Par ailleurs, ces poèmes rimés rendent la profession accessible, dans sa forme la plus populaire, à des aveugles comme à des personnes illettrées.

Les tableaux, eux, s'adressent à un public de néophytes qui veut pouvoir appliquer la méthode rapidement sans avoir besoin d'accumuler des années de pratique. Exhaustifs, ils sont conçus pour être consultés et non mémorisés. Ils révèlent également les mécanismes logiques et la cohérence interne des méthodes horoscopiques qui apparaissent ainsi accessibles à tous et non plus réservés à des «magiciens».

21. Ruli Jushi 2010: 107. 


\section{Tentatives d'institutionnalisation académique}

Ces tentatives d'adaptation des savoirs et pratiques divinatoires aux savoirs modernes, compris comme scientifiques, nourrissent une ambition de reconnaissance institutionnelle et académique. En effet, contrairement à l'Inde où l'astrologie, enseignée dans les universités et les collèges sanskrits, est pleinement établie comme discipline de l'enseignement supérieur ${ }^{22}$, les arts mantiques sont exclus du système éducatif taïwanais. Aujourd'hui à Taïwan, les personnes intéressées par les arts divinatoires se forment principalement dans des cours collectifs donnés par des spécialistes, dans le cadre de cours privés, d'associations de loisirs ou d'associations étudiantes, ou bien seules, en autodidactes, avec des manuels et l'aide éventuelle d'un ami connaisseur. Pour les réformateurs de l'horoscopie, ce mode de transmission des connaissances constitue un premier pas vers la formation d'une discipline universitaire. Leur démarche scientifique et de diffusion du savoir au plus grand nombre vise en effet à engager les études du destin dans la voie de l'académisme, c'est-à-dire à intégrer le monde de la recherche et de l'enseignement supérieur.

Ruli Jushi s'appuie ainsi sur l'exemple de la médecine chinoise pour revendiquer l'institutionnalisation académique de l'horoscopie. En effet, parmi les «cinq arts » traditionnels chinois (wushu $)^{23}$, seule la médecine chinoise s'est développée en un système scientifique et a été intégrée dans les institutions académiques modernes ${ }^{24}$. Selon Ruli Jushi, l'horoscopie moderne devrait être reconnue comme une branche de la philosophie de la vie (shengming zhexue). Mais il a conscience qu'une telle institutionnalisation ne sera possible que

22. Guenzi 2004.

23. Les wushu désignent les techniques taoïstes de longévité ( $\operatorname{shan} 山)$, la médecine ( $y i$ 醫), les techniques de calcul du destin (ming 命), la divination ( $b u$ 卜) et la physiognomonie (xiang 相).

24. Sur l'institutionnalisation et la transmission de la médecine traditionnelle chinoise en Chine continentale, voir Marié 2011. Jusqu'au début du Xx ${ }^{\mathrm{e}}$ siècle, la transmission de maître à disciple était le principal mode d'enseignement de la médecine traditionnelle chinoise, fondé sur la relation élective, personnelle et morale entre un maître et son élève, le partage de la vie quotidienne et des voyages, l'observation, l'étude intensive et la mémorisation des ouvrages classiques. À partir des années 1950, l'enseignement de masse organisé dans les universités de Chine continentale se caractérise au contraire par l'apprentissage collectif, la mobilité des étudiants, la standardisation des programmes et la faible intrusion de l'enseignement dans la vie personnelle des étudiants. À l'instar de l'essor des manuels de divination à Taïwan, la standardisation de l'enseignement à l'université «a conduit à un tri dans les matières transmises et à un abandon de la lecture et de l'étude des classiques médicaux au profit de celles de manuels composés dans ce cadre nouveau» (Obringer 2011: 15). 
si des professeurs ou des chercheurs qui possèdent déjà une reconnaissance académique entreprennent de fonder des programmes de formation élaborés à partir des conceptions rationnelles de l'«école moderne».

Ruli Jushi fonde ses espoirs sur le fait que les héritiers des lettrés de l'époque impériale qui se passionnaient pour la divination ont intégré en grand nombre le monde de la recherche et de l'enseignement supérieur et perpétuent ce loisir en marge de leur activité professionnelle. Ainsi, des professeurs de littérature expliquent les techniques divinatoires pour illustrer un cours sur le Livre des Mutations, ou pratiquent la méthode de dissection des caractères (cezi) pour conseiller les élèves sur leur orientation en dehors des cours. Certains professeurs médiatiques jouent le rôle de conseiller du prince, tel Wu Zhangyu, professeur au département de religion de l'Université Catholique Furen, considéré comme le conseiller en horoscopie du président Ma Yingjiu, et qui expose régulièrement ses analyses politiques et économiques dans les médias. Dans le monde de la recherche, l'historien Ho Peng Yoke a découvert, à l'occasion de ses propres travaux sur l'histoire des arts divinatoires, que des intellectuels de Taïwan, HongKong et des communautés chinoises de Malaisie et Singapour, perpétuent en privé des pratiques et un savoir élitistes. Ainsi, pour son travail sur la méthode des «huit signes», Ho a bénéficié de l'expertise du professeur Ho Ping-ti ${ }^{25}$.

Cependant les chercheurs, craignant les accusations de superstition, restent discrets sur leurs pratiques et ne sont pas prêts à les intégrer dans leurs activités académiques. Ho Peng Yoke raconte comment il a appris, aux détours de conversations, l'intérêt de nombreux intellectuels pour la méthode liuren, réputée particulièrement difficile:

Professor Lao Siguang is a cousin of Professor Lao Kan. He informed me that in the early 1930s, when the two cousins both lived in Peking, their parents invited an expert to come to their home to teach them the art of Liuren and the Ziping method of fate-calculation. Although I knew professor Lao Kan very much earlier, I had never heard about his knowledge in this field. I was only aware of his reputation as an eminent Chinese scholar. This example goes to show again that Liuren is a living art, although it is seldom openly practiced or talked about ${ }^{26}$.

Ainsi, le cloisonnement entre le monde universitaire et celui des praticiens constitue un obstacle à l'institutionnalisation académique souhaitée par les partisans de l' «école moderne». En effet, la recherche académique sur les arts divinatoires existe mais elle se limite principalement à une approche historique, la seule considérée comme acceptable, et exclut toute dimension

25. Cet historien sino-américain, membre de l'Academia Sinica, a été le premier président de l'Association of Asian Studies né en Asie.

26. Ho 2003: 137. 
contemporaine et pratique. Les anthropologues et sociologues taïwanais qui étudient les pratiques divinatoires depuis les années 1990 reconnaissent que seule une longue expérience permet de maîtriser véritablement ces techniques. Mais la plupart d'entre eux refusent de s'y impliquer de peur d'être soupçonnés de perdre tout recul et de se mettre à «y croire». Quant aux praticiens, détenteurs de ces savoirs, ils se montrent incapables de produire des ouvrages de référence fiables. Ainsi, l'entreprise de rationalisation menée par les praticiens de la tendance moderniste n'a jusqu'ici mené à aucun projet collectif de normalisation ni de publication de traités de référence.

L'histoire de la transmission des savoirs divinatoires de la Chine républicaine des années 1930 à la société taïwanaise contemporaine met en évidence le passage d'un mode traditionnel d'enseignement de maître à disciple à un enseignement ouvert à tous forgé sur un modèle universitaire. Mais cette transition inachevée place les arts divinatoires dans un entre-deux inconfortable ${ }^{27}$. Ayant échappé au contrôle étroit que permettait d'exercer le mode de transmission de maître à disciple, ils ne bénéficient pas pour autant de la cohésion que procure une reconnaissance académique officielle. Ainsi, la vulgarisation des arts divinatoires qui se traduit par une plus grande diversité des parcours des praticiens, favorise les clivages entre ceux qui se revendiquent comme les héritiers de lignées de maîtres, et les nouveaux praticiens. Paradoxalement, elle favorise également la multiplication des écoles de pensée qui visent précisément à pallier le manque de prestige et de légitimé de traditions récemment constituées. Et les «modernisateurs » qui militent pour une standardisation des savoirs mantiques ne constituent aux yeux de leurs détracteurs, qu'une école supplémentaire.

En Chine continentale, depuis quelques années, des signes comparables d'une ouverture au grand public des savoirs divinatoires et d'une volonté des praticiens d'uniformisation de la profession commencent à apparaître dans les grandes villes. En effet, après les persécutions de la Révolution Culturelle, les politiques religieuses ont été progressivement assouplies, surtout à partir des années 2000 ,

27. Même la médecine traditionnelle chinoise, dont l'enseignement est pourtant institutionnalisé, n'échappe pas à cette dichotomie. Ainsi, dès les années 1950 en Chine continentale, des praticiens «déclaraient que la modernisation de l'enseignement rompait la continuité de la médecine chinoise en tant que tradition vivante et ils suggéraient une valorisation plus marquée des sources classiques et un retour aux méthodes d'enseignement traditionnelles» (Marié 2011: 8). Aujourd'hui encore, la relation de maître à disciple n'a pas disparu et des professeurs forment des disciples (tudi 徒弟) à côté de leurs étudiants (xuesheng 學生) de l'université. Face à un modèle de transmission de la médecine chinoise par l'université, qui a montré ses limites, on assiste même à la tentative, depuis les années 1990, de revalorisation de l'enseignement maître-disciple (Obringer 2011: 16). 
et les pratiques de divination sont aujourd'hui largement tolérées. À Pékin par exemple, des praticiens professionnels militent pour la reconnaissance des arts mantiques, notamment sur le modèle «para-universitaire» des études nationales ( gиохие), qui offre une légitimité institutionnelle tout en permettant des activités lucratives d'enseignement. Les publications sur les arts divinatoires à destination du grand public connaissent un essor comparable à celui qui a marqué Taïwan au milieu des années 1990.

En contraste avec cette évolution, je propose de montrer comment, dans une ville de moindre importance comme Kaifeng, dans le Henan, la formation de praticiens amateurs s'accomplit sur le mode de la transmission de maître à disciple. Il n'y a à Kaifeng ni librairies spécialisées ni cours sur les arts divinatoires. L'accès aux savoirs divinatoires, s'il n'exclut pas totalement les sources écrites, passe principalement par l'enseignement oral et la relation personnelle entre le maître et son élève.

\section{Parcours initiatiques et récits légendaires en Chine continentale}

L'un des plus anciens praticiens de la ville m'a raconté les époques tourmentées qu'ont traversées les devins de Kaifeng: «Sous la République, beaucoup de personnes pratiquaient ouvertement la divination malgré les interdictions et les accusations de superstition. L'interdiction a perduré après la Libération mais les gens ont continué à exercer chez eux.» La violence de la Révolution Culturelle a entraîné un coup d'arrêt des pratiques, même clandestines. Les rares livres de divination qui circulaient à l'époque ont été brûlés. Mais la transmission n'a pas été totalement interrompue car la plupart des praticiens s'appuyaient sur un savoir oral et ne possédaient aucun livre.

Dans la suite de cet article, j'expose le cas de deux praticiens amateurs de Kaifeng. J'examine tout d'abord leur parcours d'apprentissage, leurs récits et la façon dont eux-mêmes m'ont transmis leur expérience et leur savoir pour ensuite dégager les principes et mécanismes qui régissent le mode de transmission de maître à disciple.

Lorsque j'ai rencontré M. Liu à Kaifeng en 2009, il venait de créer, avec un groupe d'amis amateurs des arts divinatoires, un cabinet de consultation à proximité de l'Université du Henan pour toucher une clientèle d'étudiants. Il m'a ainsi raconté comment il s'est initié à la divination.

Né en 1959, diplômé d'une université technique au début des années 1980, ses origines paysannes et son manque de relations ne lui ont pas permis de trouver un travail ni de s'engager dans l'armée. C'est à cette époque qu'il a commencé à s'intéresser aux «livres anciens, le genre de livres écrits en caractères non simplifiés » que lui a prêtés un ami, parmi lesquels les classiques 
du confucianisme ainsi qu'un ouvrage de physiognomonie, le Mayi shenxiang (Physiognomonie divine de Mayi), qui l'a particulièrement intéressé: «Je lisais jusqu'à ce que je m'endorme. Et quand je me réveillais, je lisais de nouveau. Puis je me suis demandé si ce que je lisais était juste ou pas, si on pouvait l'appliquer. J'ai alors décidé de voyager pour mettre ce livre en pratique.»

Ainsi, de 1983 à 1984, Liu a entrepris une sorte de voyage initiatique afin «d'élucider si le Mayi shenxiang possède un véritable caractère scientifique ou bien s'il ne s'agit que de superstitions qui doivent être rejetées en bloc». Il s'est d'abord rendu à Jinan, dans le Shandong. Pendant l'été, il s'est installé dans le parc de la Montagne aux Mille Bouddhas et a proposé ses services gratuitement. Dans ce parc, il a rencontré des praticiens originaires de toute la Chine qui lui ont enseigné d'autres techniques. Il est ensuite revenu dans le Henan, à Huaiyang, réputé pour ses spécialistes du Livre des Mutations. Pendant six mois, il s'est instruit auprès des anciens qui lui ont enseigné la méthode des «huit signes». Mais Liu a trouvé cette technique trop compliquée et préfère la physiognomonie et la chiromancie qui sont certes moins précises, mais permettent d'identifier la situation d'un individu en un seul coup d'œil. Liu a poursuivi son voyage dans le nord-est, à Pékin et à Qingdao dans le Shandong.

Au début de son voyage, Liu ne se faisait pas rémunérer pour ses services. Puis il a accepté les cadeaux, les invitations à manger et les propositions de logement. Il a ainsi réussi à vivre pendant son périple grâce à l'aide des personnes qu'il rencontrait.

À cette époque, j'ai rencontré beaucoup de bienfaiteurs (guiren), partout les gens m'ont compris et m'ont aidé. J'expliquais que mon souhait le plus cher était que les connaissances et la sagesse du Livre des Mutations puissent bénéficier au peuple. Je voulais comprendre les malheurs du peuple dans l'espoir d'alléger son fardeau. Par exemple, je suis allé plusieurs fois au Taishan ${ }^{28}$ et pendant mes séjours, je n'ai jamais payé aucun repas.

Après deux ans de voyage, Liu est rentré à Kaifeng. Malgré son expérience, il n'a pas réussi à travailler comme devin car à cette époque, explique-t-il, l'État ne soutenait pas ce genre d'activité. Mais le plus important pour lui, est que ce voyage lui a permis de découvrir son pays: «En mettant le Mayi shenxiang en pratique, j'ai compris les caractéristiques de la société chinoise.» L'expérience acquise au cours de ce voyage initiatique est une composante essentielle de la pratique des arts divinatoires: "Aujourd'hui, il y a beaucoup de livres mais la plupart ne valent rien. C'est l'expérience qui compte.» Ainsi, même si son intérêt pour la divination a été suscité par un livre, c'est le voyage et les multiples

28. Une des cinq montagnes sacrées du taoïsme, située dans la province du Shandong. 
rencontres qu'il a rendues possibles qui ont nourri son apprentissage des arts mantiques ${ }^{29}$.

Liu m'a également raconté l'histoire légendaire de la transmission du Mayi shenxiang:

Le Mayi shenxiang a été écrit par Damo ${ }^{30}$. C'était un homme très intelligent, capable d'analyser les dix mille choses et les dix mille êtres mais il sentait qu'il lui manquait quelque chose dans sa compréhension des hommes. Il a alors décidé de se retirer pendant neuf ans dans une grotte au Jiuzhushan pour méditer. Il y a écrit un livre qui, à cette époque, n'avait pas encore de titre. Il a ensuite voulu transmettre ce livre mais ne rencontrait que des gens médiocres et sans discernement. Un jour, il a fait la connaissance de Chen Xiyi, un homme très pauvre, vêtu de haillons, qui a compris la sagesse de cet enseignement. Damo a reconnu en lui son successeur et lui a transmis le livre. Chen Xiyi s'est alors mis à pratiquer la physiognomonie et a acquis une telle réputation qu'il a été surnommé «l'Immortel en haillons» (de chanvre, mayi). Un jour, Mayi a rencontré Liu Bang avant que celui-ci ne devienne empereur ${ }^{31}$. Il a examiné son visage et lui a prédit un destin extraordinaire. Mais à l'époque, personne n'a fait attention à ses paroles. Lorsque Liu Bang est devenu empereur, il a convoqué Mayi pour savoir comment il avait réussi à faire une prédiction aussi juste. Mayi lui a alors parlé du livre qu'il avait reçu de l'ancêtre fondateur Damo. Par la suite, il est probable que Liu Bang des Han ait intégré le livre dans les livres-trésors de l'Empire sous le titre de Mayi shenxiang. Voilà l'histoire de l'origine du Mayi shenxiang.

Liu semble considérer cette légende de manière littérale. Les nombreuses incohérences qu'elle recèle montrent qu'il n'a jamais cherché à approfondir ou à confirmer ce récit par des recherches ou des lectures.

Chen Tuan (877-989), alias Chen Xiyi, est en effet l'inventeur légendaire de la tradition de la physiognomonie. On lui attribue le traité de physiognomonie Shenxiang quanbian (Guide complet de physiognomonie divine) ${ }^{32}$, dont le Mayi shenxiang est une version contemporaine simplifiée. Selon la légende, Chen Tuan aurait reçu la tradition de l'immortel taoïste Lü Dongbin et d'un

29. L'expérience de Liu est typique du mode de vie itinérant et de l'univers de la figure populaire du jianghu 江湖 (littéralement «lacs et rivières»), ces chevaliers errants, moines, mendiants, devins ou vagabonds, au statut ambivalent: d'un côté, ils véhiculent l'image utopique d'individus libres, jouissant de pouvoirs surhumains et dont la réputation se forge sur le mode de la rumeur et de la légende; d'un autre côté, ils évoluent aux marges de la société, comme l'exprime un autre sens, péjoratif, de jianghu qui signifie «charlatan».

30. Le moine Bodhidharma dont il sera question plus loin.

31. Le futur empereur Gaozu 高祖 (256 ou 247-195 av. J.-C.), fondateur de la dynastie des Han.

32. Sur les légendes autour de Chen Tuan et les traités de physiognomonie qui lui sont attribués, voir Kohn 1986 et 1988. 
obscur taoïste nommé Mayi dans une grotte du Huashan. Certains passages du Shenxiang quanbian sont aussi attribués au moine indien Bodhidharma (appelé Damo en chinois), fondateur semi-légendaire du bouddhisme Chan en Chine et qui serait arrivé d'Inde en 470. Damo est connu pour avoir inventé une méthode de médiation appelée «contemplation du mur» (biguan) qu'il aurait pratiquée pendant une retraite de neuf ans. En 984, Chen Tuan reçut de l'empereur des Song Taizong (939-997) le nom de Chen Xiyi pour l'avoir reconnu en tant que futur empereur et avoir annoncé que l'Empire serait en paix lorsqu'il accéderait au trône.

Les éléments historiques dont nous disposons font remonter la tradition de la physiognomonie aux Cinq Dynasties (907-960), période à la fin de laquelle vécut Chen Tuan, dont les dons pour la physiognomonie sont attestés dans de nombreuses sources. Le Shenxiang quanbian, lui, a été compilé au début des Ming (1368-1644) par Yuan Zhongche (1367-1458), physiognomoniste à la cour impériale. Le père de Yuan Zhongche, Yuan Liuzhuang (1335-1410), fonctionnaire célèbre également pour ses talents de physiognomoniste, aurait reconnu le futur empereur des Ming Yongle (1360-1424) avant que celui-ci ne monte sur le trône.

Il est intéressant de constater que le récit de Liu mélange des personnages historiques et légendaires mais également des personnages légendaires entre eux. Liu présente Mayi et Chen Tuan comme une seule et même personne, une confusion qui apparaît dans de nombreuses légendes. En revanche, Liu ajoute un personnage à la légende en donnant le rôle du fondateur de la tradition au moine Damo. Il remplace également l'empereur Taizong des Song, que Chen Tuan a reconnu comme futur empereur, par Liu Bang, le fondateur de la dynastie Han qui a pourtant vécu un millénaire auparavant ${ }^{33}$. Par ailleurs, prédire le destin impérial des futurs empereurs est la marque des spécialistes de physiognomonie tout au long de l'histoire et il n'est guère étonnant qu'un tel exploit soit attribué à la fois à Chen Tuan et à Yuan Liuzhuang. Enfin, des éléments empruntés à différentes légendes sont intégrés à la trame générale de la légende de Chen Tuan. Ainsi, il est question d'une montagne, le Jiuzhushan, situé dans la province du Guanxi dans le récit de Liu, et du Huashan de la province du Shaanxi dans la légende plus classique de Chen Tuan. De même, il semble que, dans le récit de Liu, la légende de la grotte soit tirée de l'histoire de Mayi alors que les neuf ans de méditation font référence à celle de Damo.

33. Le lien avec Liu Bang n'est cependant pas fortuit. Le Shiji史記 rapporte en effet qu'un physiognomoniste avait prévu la montée en puissance de Liu Bang et lui donna la main de sa fille, la future impératrice Lü qui régna de -188 à -180 av. J.-C. (Chavannes, II : $325,327-330$ ). 
La véracité historique de ce récit importe moins que le fait qu'il puisse être partagé entre amis, rassemblés autour d'un intérêt commun pour la divination: il a été transmis à Liu dans un parc de Qingdao lors de son périple et lui-même m'en a fait part lors de nos discussions dans le parc à proximité de l'Université du Henan.

Le cas de M. Tang, frère aîné d'une de mes informatrices à Kaifeng, permet de retracer le processus de transfert de savoirs divinatoires sur trois générations. Tang m'a en effet enseigné les différentes techniques qu'il a lui-même reçues de son maître et qu'il utilise couramment dans les petites et les grandes affaires de la vie, pour lui-même ou pour conseiller des amis. Lors de mes séjours à Kaifeng, il venait ainsi régulièrement me rendre visite le soir, après son travail, et nous nous installions à la table de la salle à manger pour étudier, un papier et un crayon sous la main. Lors de ces rencontres, ravi de constater mon intérêt et de trouver quelqu'un digne de recevoir cet enseignement, Tang a endossé consciencieusement le rôle de professeur. Deux ans auparavant, il avait tenté d'initier sa belle-sœur mais celle-ci, «seulement diplômée du collège», n'avait jamais persévéré. Nos discussions se poursuivaient également lors de promenades dans Kaifeng.

Né en 1955 à Kaifeng, Tang est diplômé du lycée et travaille aujourd'hui dans une entreprise pharmaceutique. Comme dans de nombreux cas, ce sont des circonstances malheureuses qui l'ont poussé à s'intéresser à la divination. Au début des années 1980, Tang occupait un emploi de chauffeur dans une entreprise de transport. En cette période d'ouverture économique, un de ses amis a décidé d'ouvrir une usine. Tang s'est associé avec lui et a récolté 40000 yuans auprès de sa famille. Malheureusement, l'entreprise a fait faillite et l'ami a été arrêté pour activités illégales. Pour Tang, la honte de ne pouvoir rembourser sa famille a été insupportable. Il a démissionné et quitté Kaifeng. Dans le foyer de travailleurs où il s'est installé, il a rencontré un vieux monsieur auquel il a confié ses problèmes d'endettement. Ce dernier, trop fatigué pour se déplacer, lui a alors proposé d'aller de sa part au Jiangxi pour affaires contre une rémunération avec laquelle il pourrait commencer à rembourser ses dettes. Tang s'est donc rendu dans le Jiangxi et a profité du voyage pour visiter le Jiuhuashan, l'une des quatre montagnes sacrées du bouddhisme en Chine.

Alors qu'il logeait dans un refuge au pied de la montagne, il a rencontré un moine taoïste auquel il a raconté ses problèmes. Le lendemain, pendant l'ascension, le moine lui a prédit qu'il arriverait à rembourser ses dettes en trois ans et a offert de l'aider. Tang a ainsi passé deux semaines au Jiuhuashan pendant lesquelles le maître lui a enseigné la technique meihua yishu (numérologie de la fleur de prunier) tout en lui enjoignant de ne pas révéler ce savoir précieux à n'importe qui. Sur les conseils du moine, Tang s'est ensuite lancé dans la 
restauration et de bonnes affaires lui ont permis de rembourser ses dettes en deux ans. Cinq ans après leur première rencontre, Tang est retourné au Jiuhuashan rendre visite à son maître. Il y a séjourné un mois pendant lequel le moine lui a enseigné d'autres techniques divinatoires.

Un jour où nous pique-niquions dans un parc de Kaifeng, Tang m'a montré un genre de jeu divinatoire appelé « un dragon chinois » (Zhonghua yitiao long) ou «méthode de divination par les cartes» (pukepai suanming fa) qui se pratique avec un jeu de cartes classique, à la manière d'un solitaire. Il le tenait lui-même d'un ami qui lui a raconté que cette méthode aurait été rapportée par un militaire japonais qui aurait vécu en Chine pendant l'occupation. Celui-ci s'appelait Shanben et, comme il est mort à l'âge de 56 ans, il a été surnommé par la suite Shanben 56 (Shanben Wushiliu). Pendant son séjour en Chine, il aurait consulté un maître du Livre des Mutations pour savoir si l'attaque prévue sur Pearl Harbour réussirait. Le maître exécuta la méthode du «dragon chinois » et prédit que l'attaque sur Pearl Harbour serait un succès, mais que le Japon perdrait finalement la guerre. Shanben 56 mourut en avion pendant la guerre. Parmi les affaires qu'il avait laissées dans un coffre, on retrouva un cahier où il avait consigné la méthode transmise par le maître chinois ainsi que la prédiction sur l'attaque de Pearl Harbour. De nos jours, me raconte Tang, les Japonais croient encore beaucoup à cette méthode divinatoire.

Il est difficile d'évaluer la popularité de cette technique dont je n'ai retrouvé aucune autre trace. De brèves recherches révèlent que «Shanben 56 » n'est autre que Yamamoto Isoroku (1884-1943), dont le prénom signifie bien «56» mais qui est mort à l'âge de 59 ans $^{34}$. Commandant en chef de la Marine Japonaise pendant la deuxième guerre mondiale, il a a été responsable de l'attaque sur Pearl Harbour. Son avion a été abattu par une embuscade américaine lors d'un tour d'inspection en avril 1943. Je n'ai retrouvé aucune trace de son rôle dans la transmission de la méthode du «dragon chinois ». Cependant, quelques éléments peuvent expliquer cette légende. Tout d'abord, en tant que commandant en chef, Yamamoto était amené à planifier et donc, d'une certaine manière, à formuler des «prédictions » sur de grands événements, d'autant plus relevées par les historiens qu'elles se sont avérées justes ${ }^{35}$. De tels récits ne sont pas rares, surtout dans le domaine militaire où l'on connaît l'importance accordée aux arts divinatoires dans l'histoire chinoise. Enfin, Yamamoto était connu pour être un

34. Selon une tradition répandue, le prénom Isoroku fait référence à l'âge du père au moment de la naissance de l'enfant.

35. Ainsi, avant l'attaque sur Pearl Harbour, Yamamoto répondit de manière prophétique au Premier Ministre japonais qui l'interrogeait sur l'issue probable d'une guerre contre les États-Unis : «I shall run wild considerably for the first six months or a year, but I have utterly no confidence for the second and third years» (Gailey 1995: 68). 
joueur passionné de poker, ce qui a peut-être nourri la légende de la transmission de cette «méthode de divination par les cartes ».

La transmission du savoir de maître à disciple revêt des formes diverses selon qu'elle s'établit entre spécialistes religieux ${ }^{36}$, entre praticiens professionnels ${ }^{37}$, ou même entre l'instigateur d'un mouvement religieux et ses adeptes ${ }^{38}$. J'expose ici une forme peu institutionnalisée du lien d'enseignement qui se noue entre un spécialiste et un amateur ou entre deux amateurs. Les différents principes que révèlent les exemples de Liu et de Tang, ainsi que ma propre expérience d'apprentissage, me semblent néanmoins communs au mode de transmission orale de savoirs traditionnels dans le cadre d'une relation personnelle.

\section{Les mécanismes de la transmission de maître à disciple}

\section{La construction d'une relation privilégiée}

La transmission s'accomplit au cours de la construction d'une relation privilégiée entre la personne qui enseigne et celle qui apprend. Cette relation est tout d'abord fondée sur une «affinité prédestinée» (yuanfen), favorisée par le voyage et l'éloignement géographique initial des deux personnes. Plus les personnes viennent de loin, plus le yuanfen sera considéré comme fort. De même que Tang était destiné à rencontrer son maître au Jiuhuashan, il ne cesse de répéter que nous nous comprenons et communiquons selon une affinité prédestinée (youyuan). Si ce lien privilégié fait défaut, la transmission s'arrête d'elle-même. Ainsi, Tang a essayé d'enseigner les techniques de divination à sa belle-sœur mais, selon ses dires, celle-ci n'était pas assez intelligente et n'arrivait pas à suivre. Il est en effet souvent sous-entendu que les rencontres prédestinées ont lieu entre des personnes qui sortent du commun.

L'apprentissage s'inscrit également dans la durée. Tang a ainsi pris soin de répartir son enseignement dans le temps. Ce n'est que petit à petit qu'il m'a révélé les différentes méthodes qu'il connaissait. À la fin d'une sortie ou d'une séance de travail, il m'annonçait, comme pour me tenir en haleine: «La

36. Chez les moines taoïstes par exemple, le lien de maître à disciple est fondé sur le modèle lignager et est assimilé à la relation père-fils. Voir Herrou 2005, Goossaert 2009.

37. Pour une description de la formation de géomanciens professionnels auprès de maîtres dans un village du Sichuan dans les années 1990, voir Bruun 2003: 118-127.

38. L'histoire de l'homme qui a lancé un mouvement messianique au début des années 1980 dans le Hunan en déclarant être le prochain empereur de Chine, ressemble en de nombreux points aux expériences de Liu et Tang: mise à l'épreuve dans l'adversité, établissement de liens privilégiés avec les futurs adeptes, quête de connaissance dans les montagnes sacrées, transmission de récits légendaires. Voir Anagnost 1985. 
prochaine fois, je t'apprendrai la technique du «dragon chinois ».» Lui-même a investi du temps dans notre relation et n'a pas hésité à empiéter sur ses horaires de travail pour que nous nous rencontrions.

La transmission d'histoires ou de savoirs considérés comme précieux ou intimes se fait dans des moments particuliers. Ainsi, malgré son caractère extraverti, c'est avec une certaine pudeur que Tang m'a dévoilé les circonstances de sa rencontre avec son maître. Un jour où nous nous promenions dans un parc, nous sommes parvenus à une plate-forme circulaire au centre de laquelle trônait une pierre. Nous avons fait deux fois le tour de la plate-forme en silence puis Tang a récité un poème de Mao et un poème de Li Bai. Nous nous sommes ensuite assis sur un banc à proximité. Profitant du calme qui nous entourait, Tang m'a raconté comment il avait rencontré son maître. Sur le moment, j'ai été surprise qu'il récite ces poèmes d'un air solennel qui contrastait avec sa décontraction habituelle. Ce n'est que plus tard que j'ai fait le lien entre les poèmes et le récit de son histoire personnelle. La marche en silence et les poèmes ont contribué à créer une atmosphère et un instant propices aux confidences. Par la suite, j'ai pu observer une nette évolution dans notre relation. $\mathrm{Si}$, au début, Tang faisait preuve d'une certaine retenue, ma constance dans mon désir d'apprendre l'a rassuré. Nous nous sommes alors retrouvés de moins en moins autour de la table de travail et de plus en plus lors de sorties avec des amis. La dimension ludique des méthodes divinatoires telle qu'elle apparaît notamment dans le jeu de cartes $\mathrm{du}$ «dragon chinois » n'en diminue pas le sérieux. Au contraire, les loisirs et le temps passé ensemble renforcent l'amitié et créent des circonstances favorables à la transmission et à la pratique entre personnes de confiance.

\section{Une transmission orale et secrète}

Une autre caractéristique du processus de transmission est l'importance accordée à l'oralité. Ni Liu ni Tang n'ont cherché à vérifier dans des livres les légendes attachées aux techniques qu'ils ont apprises oralement. Ils ne se soucient pas non plus d'approfondir leur savoir en recherchant les ouvrages dont il est issu et qui sont pourtant faciles à trouver de nos jours. Ainsi, de même que Liu ignore l'existence du Shenxiang quanbian, Tang ne semble jamais avoir eu le Meihua yishu entre les mains et n'a jamais entendu parler de Shao Yong, personnage pourtant célèbre de l'histoire chinoise à qui l'ouvrage est attribué.

Tang entretient même une sorte de défiance vis-à-vis de l'enregistrement écrit. Ainsi, lorsqu'il m'a enseigné la méthode du «dragon chinois », il a refusé que je prenne des photos ou des notes. Il ne concevait pas que je puisse écrire en même temps qu'il parlait et s'arrêtait dès que je prenais mon crayon. De plus, comme il voulait contrôler le processus de démonstration, ces pauses le 
coupaient dans son élan: «Regarde d'abord, tu écriras ensuite! C'est mieux que je t'explique d'abord oralement (yanchuan bijiao hao).»

Enfin, les dispositifs mnémotechniques, les symboles et les supports tels que le jeu de cartes facilitent la transmission orale. Il est ainsi beaucoup plus facile de faire une démonstration de la méthode du «dragon chinois» que d'en rendre compte par écrit. De même, lors de nos promenades, Tang m'a fait travailler sur des cas de la méthode meihua yishu en traçant les chiffres et les trigrammes dans la terre battue avec son briquet.

Cette importance donnée à l'oralité est indissociable d'une autre dimension, celle du secret de la transmission. De même que son maître lui a recommandé de ne pas révéler les techniques à n'importe qui, Tang m'a expliqué qu'elles ne se transmettent qu'exceptionnellement de vive voix entre personnes de confiance et que je dois me contenter de les appliquer pour moi-même. Il décrit ainsi la valeur de son enseignement:

Ce que je t'ai appris aujourd'hui [sur la méthode liuren], personne ne voudra jamais te l'expliquer. Les gens aiment parler du Livre des Mutations, des cinq agents etc., mais ces techniques précises que l'on peut apprendre en une heure, personne ne te les enseignera. Et il faut savoir que même s'il n'a fallu qu'une heure pour t'expliquer le liuren et que les explications tiennent en une feuille, quand il s'agit d'étudier soimême, on peut y passer trois ans.

Un incident m'a un jour fait prendre conscience de la façon dont le mystère est entretenu autour des pratiques de divination. Alors que nous nous promenions avec une amie de Tang, celle-ci nous a fait part de son inquiétude au sujet de son fils qui s'apprêtait à passer les concours d'entrée au lycée. Tang m'a alors proposé, comme exercice, de calculer avec la méthode liuren si le fils pourrait entrer dans un bon lycée. Encore peu entraînée, j'ai commencé à calculer à haute voix pour que Tang puisse contrôler que je ne me trompais pas. Celui-ci est alors venu rapidement s'asseoir à côté de moi en me demandant de calculer dans ma tête pour ne pas révéler la procédure. Nous avons débattu entre nous à voix basse, puis Tang a donné le résultat à la mère. L'attitude de Tang est pourtant moins sectaire et plus logique qu'il n'y paraît, du fait du paradoxe inhérent aux techniques de divination: si les calculs proprement dits peuvent être enseignés en très peu de temps, l'interprétation des résultats nécessite des années d'apprentissage et d'expérience. Faire entrevoir la logique de ces techniques sans expliciter le système de pensée dans lequel elles s'inscrivent donne une fausse image de simplicité qui dévalorise les arts mantiques et nuit à leur compréhension. 
Stéphanie Homola

\section{Risques et privilèges du maître}

Même s'il n'a jamais été question de relation officielle de maître à disciple entre nous, Tang a rapidement pris goût à son rôle de professeur. L'ignorance qu'il me prêtait dans le domaine divinatoire et mon statut d'étrangère le mettaient en effet dans une position particulièrement valorisante au regard de ses compétences, finalement assez réduites. Pourtant, ce genre de relation, qui instaure une grande différence entre celui qui sait et celui qui ne sait pas, met parfois le maître dans une situation délicate. Du fait du prestige de sa position, le maître n'a pas le droit de se tromper. Or, le risque d'erreur est d'autant plus grand que les techniques divinatoires, comme tous les savoirs qui comportent une forte composante mathématique, s'oublient facilement si elles ne sont pas pratiquées régulièrement. Ainsi, à plusieurs reprises, Tang a dû dissimuler ses hésitations ou inventer des réponses à mes questions pour masquer son ignorance.

Une anecdote m'a ainsi fait réfléchir sur la crédibilité que je pouvais accorder à son enseignement. Dans la méthode du «dragon chinois » qui se pratique avec un jeu de cartes classique, les valeurs onze, douze et treize sont attribuées respectivement au valet, à la dame et au roi. Lorsque Tang m'a expliqué cette méthode, je lui ai demandé s'il existait en Chine des jeux de cartes qui n'utilisent pas les chiffres arabes mais le système de notation numérique chinois. Dans un tel cas, la dame correspond-elle par exemple au nombre douze ? Entraîné par son enthousiasme, Tang s'est mis à m'expliquer sur un ton péremptoire qu'il y a dans le monde trois types de chiffres: les chiffres arabes, romains et chinois. Il existe en effet des jeux de cartes qui utilisent les chiffres chinois où la dame correspond au douze. Et de m'expliquer ainsi le sens de la lettre Q inscrite sur la carte de la dame du jeu que nous utilisons : «En chiffre arabe minuscule, on écrit " 12 ", et en chiffre arabe majuscule, on écrit "Q". "Q" signifie " 12 " en chiffre arabe majuscule.»

Un moment de surprise passé, cette anecdote révèle, d'une part, le dogmatisme inhérent à la position du maître et, d'autre part, remet en cause la vision souvent véhiculée par les travaux historiques sur les systèmes divinatoires, de devins «experts de leur art ${ }^{39}$. La divination est pratiquée tout autant par des praticiens professionnels et des érudits que par des amateurs plus ou moins éclairés. L'expertise se définit ainsi comme un continuum et non comme une catégorie figée et bien délimitée. Les pratiques légères, ludiques voire dilettantes de la divination sont également dignes d'intérêt en ce qu'elles donnent accès à un domaine qui échappe justement à la rhétorique de l'expertise, celui des rythmes et des usages de la vie quotidienne.

39. Ce phénomène est également constaté par D. Kim à propos des chamans coréens spécialistes de divination (Kim 2009: 62). 


\section{Conclusion}

Pour les réformateurs taïwanais, engager les arts divinatoires dans la voie de la recherche académique et de l'enseignement supérieur signifie non seulement adopter une démarche scientifique mais également rendre ces savoirs accessibles au plus grand nombre. En effet, les théories mantiques, une fois standardisées et partagées par une communauté de spécialistes, peuvent être appliquées de manière mécanique et univoque à la manière de théorèmes mathématiques. Elles peuvent donc être transmises dans des livres et expliquées par des enseignants qu'une formation sanctionnée par un diplôme reconnu par l'État rend interchangeables. Ils nourrissent ainsi le projet de passer d'un mode de transmission oral, secret et individuel, fondé sur la relation personnelle et élective entre le maître et son disciple, à un enseignement uniformisé, collectif et ouvert à tous, dans le cadre d'une institution extérieure et indépendante des individus ${ }^{40}$.

Peut-on alors parler d'une crise du modèle de transmission de maître à disciple? L'exemple des praticiens amateurs de Kaifeng montre pourtant le rôle que jouent la relation personnelle et l'oralité dans la transmission des savoirs traditionnels sur le continent. Dans ce contexte, la qualité des savoir-faire transmis importe peu : les lacunes, simplifications abusives et affabulations qui émaillent les discours des praticiens témoignent de ce qui se perd et se transforme dans ces processus oraux. C'est au contraire l'appropriation personnelle des méthodes et leur usage efficace dans la vie quotidienne qui sont valorisés.

Comme tout système d'interprétation binaire, l'opposition entre transmission orale et transmission écrite est simplificatrice et ne recoupe pas entièrement la distinction que j'ai établie entre un mode de transmission de maître à disciple et un mode de transmission de type universitaire. Ainsi, même s'ils ne sont pas destinés à être isolés de l'enseignement du maître, les écrits jouent un grand rôle dans la construction de l'identité des écoles de pensée à Taïwan. À l'inverse, le mode d'apprentissage des praticiens amateurs de Kaifeng n'exclut pas entièrement les sources écrites. Quant aux cours collectifs qui s'appuient sur des manuels, répandus à Taïwan, ils n'empêchent pas le professeur de développer des relations personnelles avec ses élèves et de poursuivre son enseignement par d'intenses discussions lors de sorties ou loisirs communs.

Il me semble néanmoins possible de dire que l'importance accordée à l'écrit dans le transfert des connaissances favorise les querelles d'experts, les controverses techniques, l'obsession de l'authenticité, la compétition et la hiérarchisation des techniques et des spécialistes. Au contraire, la primauté de

40. Pour reprendre la distinction établie par l'anthropologue chinois Fei Xiaotong 費孝通 (1910-2005), ce changement de paradigme peut être interprété comme le passage d'un mode d'organisation sociale «différentiel» à un mode «organisationnel». Voir Fei 1992. 
l'oralité met la relation humaine au centre d'un processus de transmission qui lie le transfert de connaissances à la rencontre, favorisée par le voyage et la proximité des montagnes sacrées, et à l'intimité qui naît du partage des expériences et des récits légendaires. Ainsi, davantage que les expressions «maître/disciple», employé principalement dans le cas de praticiens professionnels ou de spécialistes religieux, ou «professeur/élève», utilisé dans le cadre des cours de divination à Taïwan, c'est le vocabulaire de l'amitié qui prédomine dans les liens qui se nouent entre praticiens amateurs.

\section{BIBLIOGRAPHIE}

AвE, Taizan 阿部泰山 (1968). Shenmi shengxue tianwen ziwei yunmingxue 神秘聖學 天文紫微運命學 (Étude du destin selon la mystérieuse et sainte astronomie de la Sublimité pourpre). Taipei, Guang hongwen.

ANAGNost, Ann (1985). «The Beginning and End of an Emperor : a Counterrepresentation of the State». Modern China, vol. 22, n 2: 147-176.

BAI,Huiwen 白惠文 (1936). Mingxue sucheng 命學速成 (Cours accéléré d'horoscopie). Tainan, Xingyun ge.

Bruun, Ole (2003). Fengshui in China: Geomantic Divination Between State Orthodoxy and Popular Religion. Copenhague, NIAS Press.

Chavannes, Edouard (trad.) (réed. 1969). Les Mémoires historiques de Se-ma Ts'ien. Paris, Maisonneuve.

Duara, Prasenjit (1991). «Knowledge and Power in the Discourse of Modernity: the Campaigns Against Popular Religion in Early Twentieth-century China». The Journal of Asian Studies, vol. 50, $\mathrm{n}^{\circ} 1:$ 67-83.

Fer, Xiaotong (1948, trad. 1992). From the Soil: The Foundations of Chinese Society. Berkeley, University of California Press.

Feuchtwang, Stephan (1974). «City Temples in Taipei under Three Regimes». In Elvin, Mark et Skinner, William (dir.), The Chinese City Between Two Worlds. Stanford, Stanford University Press.

Foo, Zoe (2009). «Brief Biography of Master Xu Le Wu». In site 13voices [en ligne], 24 novembre 2009, disponible sur: http://www.13voices.com/biography-xulewu, page consultée le 28 février 2012.

Galley, Harry A. (1995). The War in the Pacific: From Pearl Harbor to Tokyo Bay. Novato, Presidio Press.

GoossaerT, Vincent (2009). «Les institutions lignagères des spécialistes religieux en Chine, $16^{\mathrm{e}}-21^{\mathrm{e}}$ siècles ». In Herrou, Adeline et Krauskopff, Gisèle (dir.), Moines et moniales de par le monde. La vie monastique au miroir de la parenté. Paris, L'Harmattan. 
GuEnzi, Caterina (2004). «Destin et divination : le travail des astrologues de Bénarès ». Thèse de doctorat: Anthropologie sociale et ethnologie, École des Hautes Études en Sciences Sociales.

Herrou, Adeline (2005). La Vie entre soi: les moines taö̈stes aujourd'hui en Chine. Nanterre, Société d'ethnologie.

Ho, Peng Yoke (2003). Chinese Mathematical Astrology: Reaching Out to the Stars. London, New York, Routledge.

KIM, David J. (2009). «Divining Capital: Spectral Returns and the Commodification of Fate in South Korea ». Thèse de doctorat: Anthropologie, Université de Columbia.

Kohn, Livia (1986). «A Textbook of Physiognomy: The Tradition of the Shenxiang quanbian». Asian Folklore Studies, vol. 45 : 227-258.

KoHn, Livia (1988). «Mirror of Auras: Chen Tuan on Physiognomy». Asian Folklore Studies, vol. 47 : 215-256.

LACKNER, Michael (2012). "The Last Stand of «Chinese Science». Yuan Shushan, Traditional Divination and Western Knowledge in Republican China». Conférence «Divinatory Traditions in East Asia: Historical, Comparative and Transnational Perspectives », Rice University, 17-18 février 2012.

Marié, Eric (2011). «Transmission et pratique de la médecine chinoise». Perspectives chinoises, $\mathrm{n}^{\circ} 3: 4-12$.

Obringer, Frédéric (2011). «La médecine chinoise et la tentation du patrimoine». Perspectives chinoises, $\mathrm{n}^{\circ}$ 3: 14-22.

RULi Jushi 如理居士 (2010). Bazi guo wuguan 八字過五關 (La méthode des huit signes). Taipei, Wuling.

SATÔ, Fumio 佐藤文泍 et SATô, Rikuryû 佐藤六龍 (1968). Eiri shibi tosî : Tôyô senseijutsu nyûmon 絵入紫薇斗数: 東洋占星術入門. (Méthode illustrée des Nombres du Boisseau de la Sublimité pourpre: introduction à l'astrologie orientale). Tokyo, Haga.

SATô, Rikuryû 佐藤六龍 et ZHANG, Yaowen 張耀文 (1975). Wushu zhanbu quanshu 五術占卜全書 (Encyclopédie sur la divination et les cinq arts). Kaohsiung, Dazhong.

Sô, Keirai 曾景來 (1939). Taiwan shûkyô to meishin rôshû 臺灣宗教と迷信陃習 (La religion et les mauvaises habitudes superstitieuses à Taïwan). Taipei, Taiwan zongjiao yanjiuhui.

Xu, Lewu 徐樂吾 (1959). Mingli rumen 命理入門 (Introduction à l'horoscopie calendaire). Taipei, Wenhua.

ZHANG, Yaowen 張耀文 (1970). Ziwei chanweilu pingzhu, wu juan 紫薇闡微錄評註, 五卷 (Commentaires sur la méthode de la Sublimité pourpre, 5 tomes). Lugang, Yiwen.

ZHONG, Yiming 鍾義明 (1995). «Préface». In Mingli yongshen jinghua pingzhu 命理 用神精華評註 (Commentaires sur les esprits dans l'horoscopie). Taipei, Wuling. 
Stéphanie Homola

\section{GLOSSAIRE}

Abe Taizan 阿部泰山
baihua 白話
baishi 拜師
bazi 八字
bendiren 本地人
biantai fengsu 變態風俗
biguan 壁觀
buqiuren 不求人
cezi 測字
Chen Tuan 陳摶
Chen Xiyi 陳希夷
Chen Yueqi 陳岳琦
Damo 達摩

dizhi 地支

Doushu quanji 斗數全集

fengshui 風水

gejue 歌訣

guiren 貴人

guoxue 國學

Ho Ping-ti (He Bingdi) 何炳棣

ji 吉

jiao 珓 ou 筊

koujue 口訣

Lao Kan 勞榦

Lao Siguang 勞思光

Li Bai 李白

Liu Bang 劉邦

liuren 六壬

lumingxue 祿命學

Lunheng 論衡

Lü Dongbin 呂洞賓

Ma Yingjiu 馬英九

Mayi 麻衣

Mayi shenxiang 麻衣神相

Meihua yishu 梅花易數

mingli 命理

mingshu 命書

minshengzhuyi 民生主義

pai 派

pochu mixin yundong 破除迷信運動

pukepai suanming fa 撲克牌算命法

qian 籤

qimen dunjia 奇門遁甲 
Ruli Jushi 如理居士

sanshi 三式

Satô Rikuryû 佐藤六龍

sha 棂

Shanben Wushiliu 山本五十六

Shao Yong 邵雍

shengming zhexue 生命哲學

Shenxiang quanbian 神相全編

shisheng guanxi 師生關係

shushu 術數

sizhu 四柱

suanming re 算命熱

taiyi 太乙

Taizong 太宗

tiangan 天干

toupai 透派

waishengren 外省人

Wang Chong 王充

Wei Qianli 韋千里

Wu Zhangyu 吳彰裕

wushu 五術

xiandai pai 現代派

$\mathrm{Xu}$ Lewu 徐樂吾

Yamamoto Isoroku 山本五十六

yanchuan bijiao hao 言傳比較好

yixue 易學

Yongle 永樂

youyuan 有緣

Yuan Liuzhuang 遠柳莊

Yuan Shushan 袁樹珊

Yuan Zhongche 遠忠撤

yuanfen 緣分

zeri 擇日

zhanbu 占卜

Zhang Yaowen 張耀文

zhanyan pai 占驗派

Zhonghua yitiao long 中華一條龍

zhu 柱

ziweidoushu 紫微斗數

Poème rimé : «Déterminer l'heure à partir du jour»

Jia ji qi jia zi 甲己起甲子

Yi geng qi bing zi 乙庚起丙子

Bing xin qi wu zi 丙辛起戈子

Ding ren qi geng zi 丁壬起庚子

Wu gui qi ren zi 戊癸起壬子. 
